\title{
ANÁLISIS DE LA ESTRUCTURA FACTORIAL DEL STAI-T EN UNA MUESTRA DE DEPORTISTAS BRASILEROS
}

\author{
Daniel Bartholomeu ${ }^{1 *}$, José Maria Montiel1 ${ }^{1}$, Afonso Antonio Machado², Fabián Javier Marín Rueda ${ }^{3}$ \\ ${ }^{1}$ Centro Universitário FIEO, Osasco - Brasil, ${ }^{2}$ Universidade Julio de Mesquita - UNESP - Rio Claro \\ ${ }^{3}$ Universidade São Francisco - USF
}

Recibido, noviembre 6/2013

Concepto de evaluación, abril 3/2014

Aceptado, mayo 9/2014

\begin{abstract}
Referencia: Bartholomeu, D., Montiel, J.M., Machado, A.A. \& Rueda, F.J.M. (2014). Análisis de la estructura factorial del STAI-T en una muestra de deportistas brasileros. Acta Colombiana de Psicología, 17 (2), pp. 123-132. DOI:10.14718/ ACP.2014.17.2.13
\end{abstract}

Resumen

Este estudio tuvo como objetivo investigar la estructura interna del State-Trait Anxiety Inventory (STAI-T) en una muestra de deportistas de Brasil. Participaron 179 sujetos que practicaban cinco deportes diferentes, con edades entre 14 y 58 años $(M=21,04 ; D T=4,21)$. El inventario evalúa la ansiedad como estado y como rasgo, pero en este estudio fue utilizada apenas la escala referente a los rasgos, compuesta por 20 ítems dispuestos en una escala Likert de cuatro puntos. Los resultados del análisis factorial confirmatorio indicaron que los ítems no se ajustaron adecuadamente al modelo originalmente establecido por el instrumento y, considerando ese resultado, se optó por realizar un análisis factorial exploratorio, el cual mostró la existencia de dos factores que explicaron el 33\% de la variancia. Esos factores fueron denominados de ansiedad presente y ansiedad ausente, con coeficientes de precisión con valores de 0,82 y 0,72 respectivamente.

Palabras clave: STAI-T, evidencia de validez, deportistas, psicología del deporte, evaluación psicológica.

\section{STAI-T FACTOR STRUCTURE ANALYSIS IN A SAMPLE OF BRAZILIAN ATHLETES}

\begin{abstract}
This study aimed to examine the internal structure of the State-Trait Anxiety Inventory (STAI-T) in a sample of Brazilian athletes. Participants were 179 subjects practicing five different sports modalities, aged between 14 and 58 years $(\mathrm{M}=21.04$; $\mathrm{SD}=4.21$ ). The inventory assesses anxiety as state and trait, but in this study only the trait anxiety scale was used which is composed of 20 items arranged in a four-point Likert scale. Results of the confirmatory factor analysis indicated the items were not properly adjusted to the instrument's original model. This exploratory factor analysis indicated the existence of two factors that explained $33 \%$ of the variance. These factors were called present anxiety and absent anxiety, with reliability coefficients of 0.82 and 0.72 respectively.

Key words: STAI-T, validity evidences, athletes, sport psychology, psychological assessment.
\end{abstract}

* Daniel Bartholomeu, Centro Universitário FIEO - UNIFIEO, Avenida Franz Voegeli, 300 - CEP: 06020-190, Tel. 55-11- 3651-9999 55-11-98385-4762.03081@unifieo.br d_bartholomeu@yahoo.com.br 


\title{
ANÁLISE DA ESTRUTURA FATORIAL DO STAI-T EM UMA MOSTRA DE ESPORTISTAS BRASILEIROS
}

\begin{abstract}
Resumo
Este estudo teve como objetivo pesquisar a estrutura interna do State-Trait Anxiety Inventory (STAI-T) em uma mostra de esportistas do Brasil. Participaram 179 sujeitos que praticavam cinco esportes diferentes, com idades entre 14 e 58 anos $(M=21,04 ; D T=4,21)$. O inventario avalia a ansiedade como estado e como rasgo, mas neste estudo foi utilizada apenas a escala referente aos rasgos, composta por 20 itens dispostos em uma escala Likert de quatro pontos. Os resultados da análise fatorial confirmatoria indicaram que os itens não se ajustaram adequadamente ao modelo originalmente estabelecido pelo instrumento e, considerando esse resultado, se optou por realizar uma análise fatorial exploratória, que mostrou a existência de dois fatores que explicaram a variância de $33 \%$. Esses fatores foram denominados de ansiedade presente e ansiedade ausente, com coeficientes de precisão com valores de 0,82 e 0,72 respectivamente.

Palavras chave: STAI-T, evidência de validade, esportistas, psicologia do esporte, avaliação psicológica.
\end{abstract}

\section{INTRODUCCIÓN}

En la década de 1970, Spielberger et. al. (1970) desarrollaron el State-Trait Anxiety Inventory (STAI), compuesto por escalas para evaluar la ansiedad rasgo y la ansiedad estado, llamadas STAI-T y STAI-S, respectivamente. Este estudio se centró en el análisis del STAI-T, que ha sido utilizado en una amplia variedad de deportes, como el baloncesto, fútbol, tenis, gimnasia, natación, lucha, entre otros (Hackfort \& Spielberger, 1989). Además de su importancia histórica, el STAI ha sido uno de los instrumentos más utilizados en la evaluación de la ansiedad en diferentes contextos, ya que su modelo teórico fue base para la construcción de muchas otras pruebas de ansiedad (Smith, Smoll \& Wiechman, 1999).

Sobre sus propiedades psicométricas, las informaciones del manual indican una adecuada confiabilidad obtenida por el método test-retest, con correlaciones que oscilaron entre 0,73 y 0,84 . Los valores alfa de Cronbach también fueron satisfactorios, oscilando de 0,83 a 0,92 . La validez concurrente fue verificada por las correlaciones con otras pruebas de ansiedad rasgo, como el Taylor's Manifest anxiety Scale y el Zuckerman's Affect Adjective Checklist (Cattell \& Scheier, 1963), y los coeficientes oscilaron entre 0,75 y 0,80 . En relación con la estructura factorial del instrumento original, los resultados han sido inconsistentes en el número de factores, lo que llevó a los autores del instrumento a realizar una revisión de su estructura. En esta nueva versión, los estudios factoriales han señalado una estructura multidimensional más coherente con dos factores, interpretados como 'ansiedad presente' $y$ 'ansiedad ausente' para la escala de rasgos - STAI-T (Schwenkmezger \& Laux, 1986).

Posteriormente, en algunos países se han llevado a cabo otros estudios sobre la estructura factorial de la prueba. Al respecto, la adaptación española del STAI-T fue realizada por Bermúdez (1977) en su tesis doctoral.
La confiabilidad varió de 0,84 a 0,87 , y el análisis factorial identificó cuatro factores, dos de ansiedad estado (presente y ausente) y dos de ansiedad rasgo (también presente y ausente), que explicaron el 68\% de la variancia. Estos resultados fueron posteriormente corroborados por Urraca (1981), también en España. En Colombia, Moreno y Copete (2005) realizaron un estudio con niños de 8 a 15 años, también con el objetivo de analizar la estructura factorial del STAI en sus versiones rasgo y estado. Los autores identificaron seis factores, denominados temor, tranquilidad, preocupación, evitación, somatización e ira. Los tres primeros se refieren a estados de ansiedad, mientras que los tres últimos, a rasgos.

Específicamente en el contexto del deporte, el instrumento ha sido empleado ampliamente para analizar, por ejemplo, sus relaciones con el desempeño atlético y con características sociodemográficas de los deportistas, además de otras variables (Abenza et. al., 2009; Aguirre-Loaiza \& Bermúdez, 2011; Gillet, Rosnet \& Vallerand, 2008). Pese a esto, los estudios sobre su estructura factorial son más escasos en este ámbito, entre los cuales se destaca el trabajo colombiano de Serrato, García y Rivera (2000) quienes elaboraron baremos para el STAI en un grupo de deportistas. Los factores de ansiedad-rasgo encontrados fueron dos, y sugirieron una ansiedad presente y una ausente (semejante a la propuesta original de Spielberger), pese a que los coeficientes de consistencia interna fueron menores de 0,50 para las dos escalas, lo cual no reveló resultados satisfactorios. En Brasil, a pesar de que el STAI-T es usado en diferentes contextos y con diversas muestras, no hay estudios que hayan analizado su estructura interna con la población de deportistas. La mayor parte de los trabajos sobre esta prueba están centrados en estudiar las relaciones entre ansiedad y otras variables, como el desempeño de los deportistas, el tiempo de recuperación de lesiones y la cantidad de entrenamientos, entre otras (Gonçalvez \& Belo, 
2007; Pesca, 2004; Wamba, Viccari, Eisenhut, Radtke Jr. \& Shigunov 2008).

Considerando que no existen estudios sobre la estructura del STAI-T en muestras de deportistas brasileros, y que el instrumento es uno de los más utilizados para la evaluación de la ansiedad, la investigación en torno a esa estructura reviste gran importancia. Además, la relevancia aumenta si se considera que los trabajos que evaluaron la estructura de esta prueba en diferentes muestras presentaron variaciones. Un ejemplo de esto es el estudio brasilero de Fioravanti, Santos, Maissonette, Cruz y Landeira-Fernandez (2006), que tuvo como objetivo investigar las características psicométricas del STAI-T en una muestra de 1.589 personas, la cual se dividió en tres grupos: estudiantes universitarios, estudiantes de enseñanza secundaria y militares. Los resultados indicaron estructuras factoriales distintas en cada una de las muestras estudiadas. En la muestra de universitarios y de enseñanza secundaria la estructura factorial fue interpretada como un factor relativo a ansiedad y otro a depresión, mientras que en el grupo de militares la estructura fue de un factor de ansiedad presente y otro de ansiedad ausente. Este estudio es importante pues muestra una diferencia clara entre las estructuras del STAI dependiendo del tipo de muestra. Esto llevó a formular la pregunta de cómo sería la estructura de la prueba al considerar una muestra de deportistas brasileros, pregunta que esta investigación trató de responder.

Debe destacarse que las divergencias en los factores, de acuerdo con el tipo de muestra, a menudo han sido identificadas en estudios brasileros con el STAI-T. Entre ellos, el trabajo de Andrade, Gorenstein, Vieira, Tung y Artes (2001), que analizó una muestra de universitarios, evidenció una estructura de dos factores (ansiedad presente y ausente) semejante al estudio de Fioravanti et. al. (2006). A su vez, Pasquali, Pinelli Jr. y Solha (1994) realizaron una investigación con personas en proceso de selección y predominantemente del sexo masculino, y la estructura identificada fue semejante a la de Fioravanti et. al. (2006) en la muestra de militares. Pese a esas diferencias observadas, no parece claro el motivo por el cual algunos tipos de muestras podrían afectar la distribución de cargas factoriales en el instrumento. De hecho, algunos ítems no diferencian bien los constructos que, a pesar de estar altamente correlacionados, dado que, comparten algunos síntomas como tensión e irritabilidad, son constructos distintos. También existen investigaciones que demuestran que el STAI-T no es un buen instrumento para diferenciar trastornos de ansiedad y depresión (Bartholomeu et. al., 2009; Fioravanti et. al., 2006; Kennedy, Schwab, Morris \& Beldia, 2001).
Considerando esas divergencias en la estructura, y que el STAI-T es un instrumento muy empleado en Brasil en trabajos desarrollados con deportistas y atletas, este estudio se propone comparar la estructura factorial obtenida en una muestra de deportistas con los resultados obtenidos en trabajos que investigaron otras muestras. De esa forma, el objetivo central fue identificar una estructura factorial para el STAI-T en una muestra de deportistas.

\section{MÉTODO}

\section{Participantes}

El estudio fue realizado con 179 deportistas de ambos sexos, de los cuales, 59,2\% eran hombres. Las edades oscilaban entre 15 y 35 años, con una media de 21,04 $(D T=4,21)$. En relación con el nivel educacional, la mayoría era universitario $(64,2 \%)$. En relación con el deporte, debe mencionarse que los participantes no eran atletas, sino que practicaban deportes frecuentemente, ya que más del $80 \%$ de la muestra lo hacía entre tres y cinco veces por semana, durante dos o tres horas por día. Se destaca que el 58,7\% de los sujetos desempeñaba actividades laborales además de practicar deportes. El estudio fue realizado en varios clubes deportivos del interior del estado de San Pablo, Brasil, y en relación con las modalidades practicadas, $25,7 \%$ se refería a fútbol, $21,8 \%$ a balonmano, $20,1 \%$ a voleibol, $14 \%$ a baloncesto, $12,8 \%$ a diferentes tipos de lucha (karate, jiujitsu, judo) y $5,6 \%$ a tenis. Pese a que los sujetos no eran atletas, $91,6 \%$ ya había participado en alguna competición en su respectivo deporte.

\section{Instrumento}

State-Trait Anxiety Inventory - STAI-T (Spielberger, Gorsuch \& Lushene, 1970). La versión para este estudio fue la que Spielberger, Gorsuch y Lushene (1970) crearon originalmente. Pese a que el instrumento fue construido con el objetivo de medir la ansiedad rasgo y la ansiedad estado, en este estudio fueron utilizados únicamente los ítems relacionados con la ansiedad rasgo, considerando que es el tipo de ansiedad menos estudiado en el contexto deportivo. Esta versión presenta 20 ítems que indagan acerca de cómo se siente la persona en relación con diversos síntomas de ansiedad. Las respuestas posibles son 1 (casi nunca), 2 (a veces), 3 (con frecuencia) y 4 (casi siempre). En la corrección, las respuestas son sumadas ítem a ítem, proporcionando un puntaje total que puede variar de 20 a 80. La puntuación en los ítems 1, 6, 7, 10, 13, 16 y 19 debe ser invertida para realizar la sumatoria final. 


\section{Procedimiento}

Los datos fueron recogidos en aplicaciones realizadas colectivamente en locales que los clubes cedieron para la investigación. Toda la recolección de datos fue realizada por dos investigadores, un psicólogo y un profesor de educación física, momentos antes de los entrenamientos deportivos en cada modalidad. Participaron en el estudio sólo los sujetos que estuvieron de acuerdo, y en el caso de los menores de edad, aquellos que estuvieron de acuerdo y cuyos padres o representantes legales también autorizaron la participación por escrito. La aplicación no demoró más de diez minutos.

\section{Análisis de datos}

Considerando el objetivo del estudio, que es verificar si la estructura de dos factores del STAI-T se mantiene en una muestra de deportistas brasileros, se realizó un análisis factorial confirmatorio, usando la versión del programa AMOS 16,0. Para ello, inicialmente se verificó que las omisiones no llegaran al 5\% del total de las respuestas, y que tampoco fueran detectados problemas de multicolinearidad o casos extremos univariados o multivariados, que pudieran afectar los resultados. Pese a eso, se observó que los valores de Kurtosis oscilaban entre $-0,925$ y 43,898 y de Skewness entre 0,366 y 5,056. Además, sólo cuatro ítems (3, 5, 14, 15) estuvieron entre los límites de -1 y 1 en Skewness y en Kurtosis, y el ítem 2 sólo en Kurtosis. Por lo tanto, los datos fueron relativamente anormales, lo que justificó la utilización del método de máxima verosimilitud para la estimación de parámetros, como lo indican Arbuckle y Worthke (1999). Para verificar el ajuste al modelo se utilizó el índice de la relación del chi-cuadrado dividido por los grados de libertad, con valor límite de 2; los índices TLI (Tucker-Lewis Index), CFI (Comparative Fit Index) y NFI (Normed Fit Index), considerando como valores adecuados aquellos superiores a 0,9; y el RMSEA (Root Mean Square Error of Aproximation), considerando un valor adecuado igual o inferior a 0,08 .

\section{RESULTADOS Y DISCUSIÓN}

La figura 1 muestra el estudio confirmatorio de la estructura del STAI-T con sus respectivos coeficientes (que deben interpretarse como los coeficientes de regresión). Las elipses representan los factores latentes; los rectángulos, las variables observadas - ítems; y los círculos, el error de cada variable observada. Las flechas de una vía indican que cada factor latente produce una respuesta a cada uno de los ítems asociados. Sin embargo, las respuestas no están determinadas únicamente por el factor, sino también por otros aspectos desconocidos (debido a errores). Las flechas bidireccionales sugieren una interrelación entre los factores. Todos los coeficientes que están en la figura 1 fueron significativos y superiores a 0,32 .

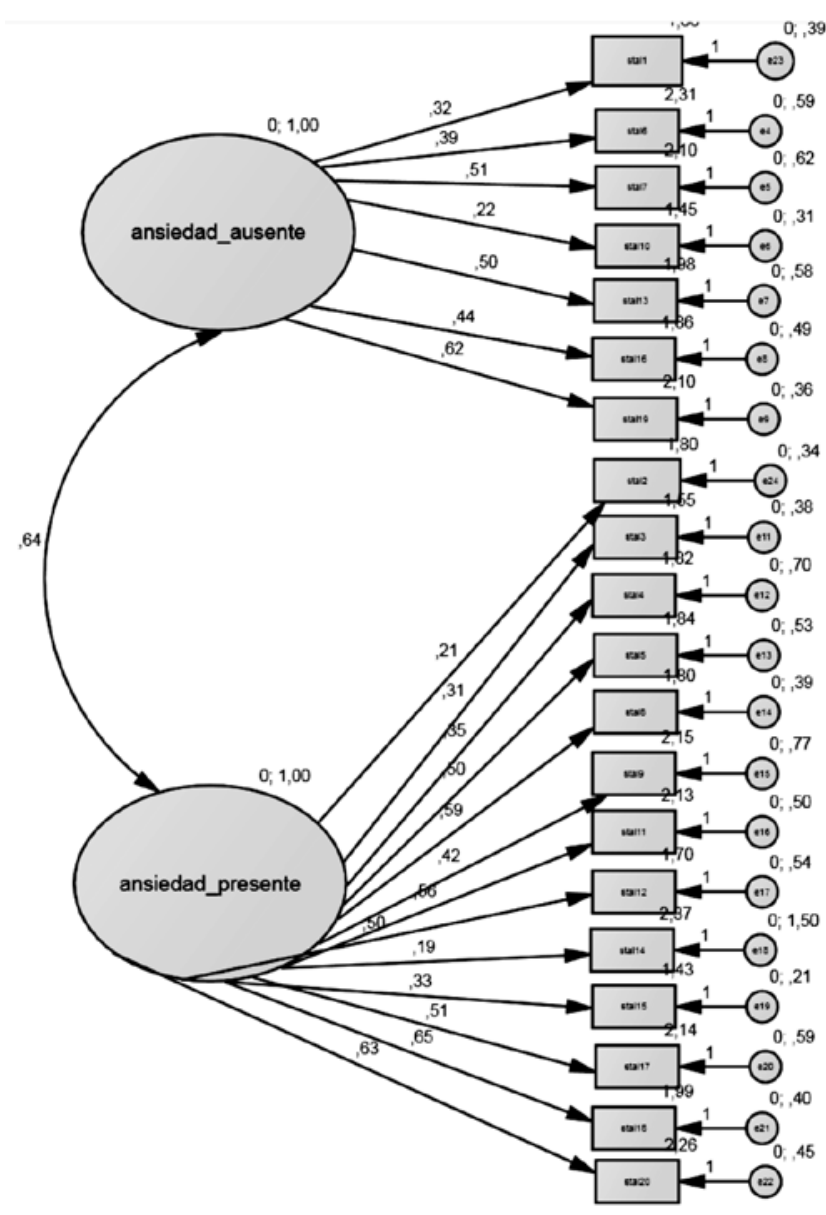

Figura 1. Modelo de dos factores del STAI-T $(N=179)$.

El análisis realizado mostró que el ajuste al modelo no fue satisfactorio, pues mostró apenas algunos valores marginales $(x 2 / g l=2,57, \mathrm{CFI}=0,62, \mathrm{NFI}=0,51$, RMSEA $=0,102$, $\mathrm{TLI}=0,58)$. Teniendo esto en cuenta, se consideró pertinente utilizar técnicas de análisis factorial exploratorio para identificar cuál sería la mejor estructura subyacente a los datos. Para realizar un análisis factorial, la previsión mínima de la muestra debe ser de cinco participantes por ítem de la escala. Considerando que el STAI-T tiene 20 ítems, serían necesarios por los menos 100 participantes para realizar el análisis (Tabachinick \& Fidell, 1996). De acuerdo con ese aspecto, el número de deportistas que conformaron la muestra cumplía con el requisito mínimo para llevar a cabo el análisis. Además de esto, deben cumplirse otros criterios para garantizar resultados válidos. Dos de esos criterios son la medida de adecuación de la muestra de Kaiser-Meyer- 
Olkin $(\mathrm{KMO}=0,85)$ y la prueba de esfericidad de Bartlett $\left(X^{2}=725,36, g l=120, p=0,000\right)$ que sugirieron la posibilidad de extraer más de un factor con los ítems del STAI-T en los deportistas, indicando buenos resultados.

Una vez hecho esto, se pasó al análisis relacionado con la cantidad de factores que deberían ser extraídos. Algunos métodos que se utilizan para este fin se presentarán a continuación. Por el criterio de Kaiser, los factores con valores propios superiores a 1 deben mantenerse. En esa circunstancia, serían cuatro los factores considerados, y ellos explicarían el 56,34\% de la variancia. En relación con el análisis anti-imagen, que indica una correlación parcial inversa entre los ítems, éste mostró que todos los coeficientes eran cero, lo que sugiere la existencia de más de un factor (Tabachinick \& Fidell, 1996). Además de estos métodos, el análisis paralelo se utiliza a menudo para verificar la cantidad de factores que deben conservarse en un análisis factorial. La validez de la estructura de los factores subyacentes a una serie de ítems debe tener valores propios mayores que los componentes paralelos derivados de datos aleatorios con el mismo tamaño de la muestra y el mismo número de ítems, y los investigadores no deben interesarse en los factores que no tienen en cuenta más que la variancia proporcionada por factores aleatorios. Con esta consideración, debe destacarse que eigenvalues reales inferiores o iguales a los eigenvalues medios aleatorios del análisis paralelo son atribuibles a errores de muestreo (Hayton, Allen \& Scarpello, 2004).

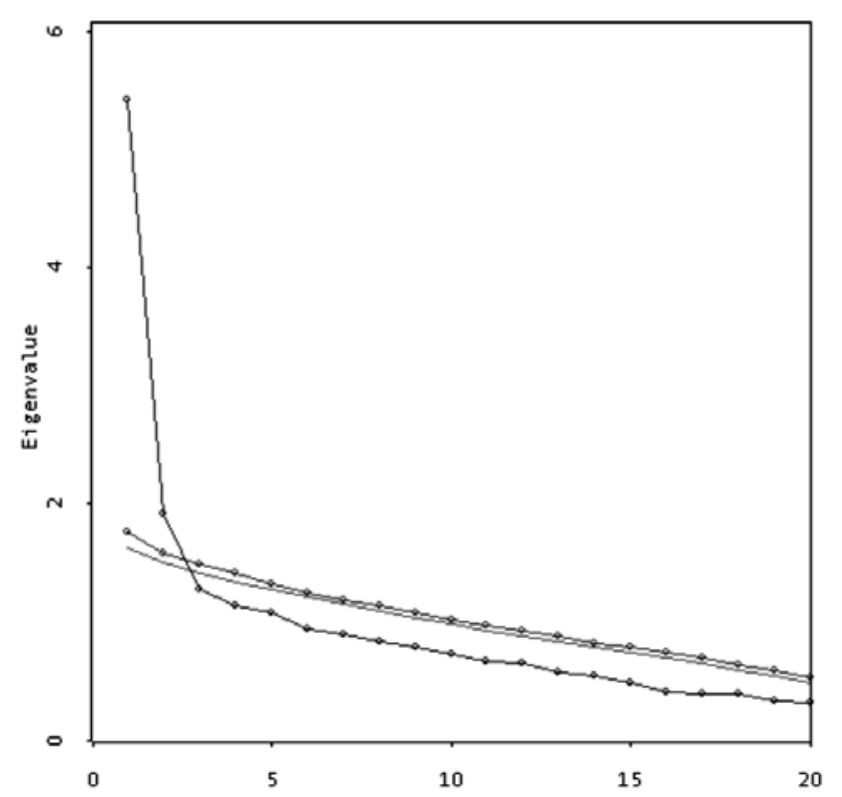

Figura 2. Gráfico de Sedimentación para los ítems del STAI-T $(N=179)$.
De hecho, encontramos que en los dos primeros factores, los eigenvalues generados por los datos reales extraídos del STAI-T tuvieron valores propios mayores que los eigenvalues generados aleatoriamente mediante el análisis paralelo, teniendo en cuenta los valores medios o del baremo 95 (el baremo 95 es más conservador, y equivale a ajustar los valores de alfa para 0,05 ). Así, este análisis confirma la necesidad de extracción de dos factores en estos datos. La figura 2 muestra el gráfico de sedimentación de los factores observados y estimados aleatoriamente por el análisis paralelo.

En la figura 2 el eje Y muestra los valores de los eigenvalues, mientras que el eje $\mathrm{X}$ es el número de factores. Según Cattell (1966), el uso de este gráfico es uno de los procedimientos para determinar el número de factores que serán extraídos de un conjunto de ítems. Como puede observarse, hay una mayor discontinuidad en la curva cuando se representa el segundo factor, lo que sugiere la posibilidad de una estructura de dos factores. Así, los datos fueron evaluados mediante el análisis de los ejes principales con rotación Quartimax y eigenvalues iguales o superiores a 1,0. La elección de este modelo estadístico ocurrió por observar la presencia de factores independientes entre sí, como también ocurrió en otros estudios con el STAI-T, lo que justifica el uso de una rotación ortogonal. Además de eso, se esperaba no utilizar una parte de la variancia de error, lo que también justificó no emplear un modelo de extracción de componentes principales. Resumiendo, decidimos mantener la configuración inicial proporcionada por el gráfico de sedimentación y por el análisis paralelo, o sea, optamos por los dos factores que estaban más apartados del resto. Los ítems con sus cargas factoriales y la variancia explicada por cada factor se muestran en la Tabla 1. En esta tabla también se muestran los eigenvalues del primer factor medio y el baremo 95, proporcionados por el análisis paralelo.

En estas condiciones restaron 16 ítems divididos en dos factores, explicando $33,54 \%$ de la variancia. En la Tabla 1 se observa que el factor 1 , con diez ítems, está bastante relacionado con la ansiedad presente y explicó el $22,46 \%$ de la variancia. A su vez, el factor 2 presenta seis ítems más relacionados con características positivas, y fue denominado 'ansiedad ausente'. Este factor explicó el $11,77 \%$ de la variancia total. A partir de estos datos se calcularon los coeficientes de consistencia interna por medio de la correlación ítem-total en cada factor. Los valores de $r$ encontrados no fueron inferiores a 0,30 en ninguno de los ítems de los dos factores, con excepción del ítem 2 ('Me canso con facilidad'), que tuvo una correlación de 0,29 . El valor 0,30 se considera aceptable para este tipo de análisis (Guilford \& Fruchter, 1978), y por más que el valor del 
Tabla 1.

Factores, saturación, h2, eigenvalues y variancia explicada observados en el análisis de los ejes principales, con rotación Quartimax, para el STAI-T $(N=179)$.

\begin{tabular}{|c|c|c|c|}
\hline & \multicolumn{2}{|c|}{ Factores } & \multirow{2}{*}{$\mathrm{h} 2$} \\
\hline & 1 & 2 & \\
\hline $\begin{array}{l}\text { 17. Ideas sin importancia a veces } \\
\text { me dejan preocupado. }\end{array}$ & 0,68 & & 0,49 \\
\hline $\begin{array}{l}\text { 18. Las decepciones me preocupan } \\
\text { tanto que no dejo de pensar en ellas. }\end{array}$ & 0,68 & & 0,51 \\
\hline $\begin{array}{l}\text { 20. Me pongo tenso(a) y me } \\
\text { perturbo cuando pienso en mis } \\
\text { problemas actuales. }\end{array}$ & 0,66 & & 0,47 \\
\hline $\begin{array}{l}\text { 11. Dejo que las cosas me afecten } \\
\text { mucho. }\end{array}$ & 0,65 & & 0,45 \\
\hline 15. Me siento deprimido(a). & 0,58 & & 0,36 \\
\hline $\begin{array}{l}\text { 8. Siento que las dificultades van } \\
\text { acumulándose y no las puedo } \\
\text { resolver. }\end{array}$ & 0,58 & & 0,39 \\
\hline $\begin{array}{l}\text { 12. No tengo confianza en mí } \\
\text { mismo(a). }\end{array}$ & 0,50 & & 0,33 \\
\hline $\begin{array}{l}\text { 9. Me preocupo demasiado por } \\
\text { cosas sin importancia. }\end{array}$ & 0,49 & & 0,24 \\
\hline $\begin{array}{l}\text { 4. Me gustaría ser feliz como las } \\
\text { otras personas. }\end{array}$ & 0,35 & & 0,13 \\
\hline 2. Me canso con facilidad. & 0,31 & & 0,10 \\
\hline 16. Estoy satisfecho(a). & & 0,55 & 0,36 \\
\hline 1. Me siento bien. & & 0,52 & 0,32 \\
\hline 19. Soy una persona estable. & 0,42 & 0,50 & 0,42 \\
\hline 10. Soy feliz. & & 0,47 & 0,23 \\
\hline 13. Me siento seguro(a). & & 0,47 & 0,29 \\
\hline $\begin{array}{l}\text { 7. Soy tranquilo(a), equilibrado(a) y } \\
\text { dueño(a) de mí mismo(a). }\end{array}$ & & 0,42 & 0,26 \\
\hline Porcentaje de variancia explicada & 22,46 & 11,07 & 33,54 \\
\hline Eigenvalue observado & 3,59 & 1,77 & \\
\hline $\begin{array}{l}\text { Eigenvalue promedio esperado } \\
\text { (Factor } 1 \text { análisis paralelo) }\end{array}$ & 1.64 & & \\
\hline $\begin{array}{l}\text { Eigenvalue esperado baremo } 95 \\
\text { (Factor } 1 \text { análisis paralelo) }\end{array}$ & 1.74 & & \\
\hline
\end{tabular}

ítem 2 haya sido más bajo, se optó por mantenerlo en la escala, teniendo en cuenta su importancia en el contexto del deporte.

También se calcularon los coeficientes alfa de Cronbach para cada factor. El factor 1 mostró un coeficiente de 0,82 y el factor 2, de 0,72. El procedimiento de las dos mitades de Spearman-Brown sugirió coeficientes de 0,84 y 0,73 para los factores 1 y 2 , respectivamente. Los valores obtenidos se pueden considerar bastante aceptables, teniendo en cuenta la cantidad de ítems en cada factor, así como la recomendación mínima para este tipo de análisis de acuerdo con la APA, AERA y NCME (1999), que es de 0,70.

Dando continuidad a los resultados obtenidos, en la Tabla 2 se presentan las cargas factoriales de todos los ítems (incluyendo aquellos con cargas factoriales mayores de 0,30 , considerado el mínimo aceptable para la inclusión del ítem en el factor), para permitir una comparación con la estructura factorial obtenida en otros estudios publicados en la literatura brasilera que se realizaron con muestras de sujetos que no eran deportistas.

Las variancias comunes encontradas en 15 ítems presentaron índices inferiores a 0,50 , es decir, por lo menos la mitad de la variancia de cada variable debe ser relacionada con el factor (Hair, Anderson, Tatham \& Black, 1995). MacCallum, Widaman, Zhang y Hong (1999) destacan que cuando la variancia común es baja, el papel del tamaño de la muestra y la 'overdetermination' (seis o siete indicadores por factor y un bajo número de factores) son aspectos importantes en la recuperación de los factores de la población, y el punto 0,50 es un buen índice para conseguir esto. Cuando las variancias comunes están consistentemente por debajo de ese valor (como es el caso de este estudio), para garantizar una buena recuperación de los factores se necesita la overdetermination de los factores, así como también muestras grandes (superiores a 100 sujetos). En este estudio, la estructura identificada fue de dos factores con por lo menos seis indicadores en cada factor, y el tamaño de la muestra superior a 100, cumpliendo de esa forma el criterio de MacCallum et. al. (1999) y sugiriendo la posibilidad de una buena estimación de los factores poblacionales.

Como se mencionó anteriormente, los datos presentados sugieren el primer factor como 'ansiedad presente', y el segundo, como 'ansiedad ausente'. Este resultado es similar al de Fioravanti et. al. (2006) con una muestra de militares, y al de Pasquali et. al. (1994) con una muestra de participantes que estaban pasando por un proceso de selección. Ya en las muestras de universitarios (Andrade et. al., 2001; Fioravanti et. al., 2006) y de estudiantes de enseñanza secundaria (Fioravanti et. al., 2006), los resultados de las investigaciones brasileras indicaron un factor de ansiedad y otro de depresión.

Es importante destacar que las investigaciones mencionadas fueron seleccionadas, ya que en Brasil son las únicas que tuvieron como propósito estudiar la estructura factorial del STAI utilizando diferentes muestras. Específicamente, en relación a Fioravanti et. al. (2006), las diferencias observadas en el estudio suscitaron la necesidad de investigar una estructura específica del instrumento para deportistas, una vez que el test es uno de los más 
Tabla 2.

Comparación de la estructura factorial de los estudios con el STAI-T*

\begin{tabular}{|c|c|c|c|c|c|c|c|c|c|c|c|c|}
\hline \multirow[b]{2}{*}{ Ítems } & \multicolumn{2}{|c|}{$\begin{array}{c}\text { Pasquali } \\
\text { (Personas en } \\
\text { proceso de } \\
\text { selección) }\end{array}$} & \multicolumn{2}{|c|}{$\begin{array}{l}\text { Andrade et. al. } \\
\text { (Universitarios) }^{* *}\end{array}$} & \multicolumn{2}{|c|}{$\begin{array}{l}\text { Fioravanti et. al. } \\
\text { (Universitarios) }\end{array}$} & \multicolumn{2}{|c|}{$\begin{array}{c}\text { Fioravanti et. } \\
\text { al. (Enseñanza } \\
\text { secundaria) }\end{array}$} & \multicolumn{2}{|c|}{$\begin{array}{l}\text { Fioravanti et. al. } \\
\text { (Militares) }\end{array}$} & \multicolumn{2}{|c|}{$\begin{array}{l}\text { Bartholomeu et. } \\
\text { al. (Deportistas) }\end{array}$} \\
\hline & 离 & 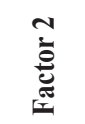 & 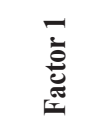 & 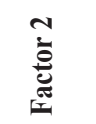 & 节 & 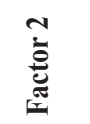 & 㐫 & 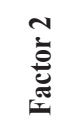 & 节 & 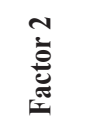 & 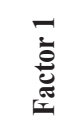 & 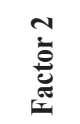 \\
\hline $\begin{array}{l}\text { 17. Ideas sin importancia a veces me } \\
\text { dejan preocupado. }\end{array}$ & 0,62 & $-0,26$ & 0,71 & 0,13 & 0,73 & 0,16 & 0,59 & 0,02 & 0,70 & 0,08 & 0,71 & $-0,20$ \\
\hline $\begin{array}{l}\text { 18. Las decepciones me preocupan tanto } \\
\text { que no dejo de pensar en ellas. }\end{array}$ & 0,57 & $-0,26$ & 0,62 & 0,35 & 0,62 & 0,24 & 0,60 & 0,11 & 0,52 & 0,10 & 0,70 & 0,20 \\
\hline $\begin{array}{l}\text { 20. Me pongo tenso(a) y me perturbo } \\
\text { cuando pienso en mis problemas actuales. }\end{array}$ & 0,56 & $-0,20$ & 0,59 & 0,27 & 0,48 & 0,33 & 0,61 & 0,11 & 0,59 & 0,09 & 0,68 & 0,15 \\
\hline 11. Dejo que las cosas me afecten mucho. & 0,51 & $-0,18$ & 0,69 & 0,22 & 0,64 & 0,26 & 0,65 & 0,08 & 0,47 & 0,08 & 0,65 & 0,14 \\
\hline $\begin{array}{l}\text { 8. Siento que las dificultades van } \\
\text { acumulándose y no las puedo resolver. }\end{array}$ & 0,60 & $-0,30$ & 0,40 & 0,42 & 0,36 & 0,40 & 0,50 & 0,18 & 0,38 & 0,13 & 0,61 & 0,35 \\
\hline 15. Me siento deprimido(a). & 0,56 & $-0,28$ & 0,31 & 0,66 & 0,34 & 0,54 & 0,42 & 0,49 & 0,20 & 0,13 & 0,61 & 0,14 \\
\hline $\begin{array}{l}\text { 9. Me preocupo demasiado por cosas sin } \\
\text { importancia. }\end{array}$ & 0,55 & $-0,25$ & 0,71 & 0,10 & 0,71 & 0,14 & 0,58 & 0,02 & 0,47 & 0,04 & 0,56 & $-0,10$ \\
\hline $\begin{array}{l}\text { 5. Pierdo oportunidades por no conseguir } \\
\text { tomar decisiones rápidas. }\end{array}$ & 0,62 & $-0,21$ & 0,44 & 0,30 & 0,35 & 0,25 & 0,34 & 0,13 & 0,36 & 0,11 & 0,55 & 0,19 \\
\hline 3. Tengo ganas de llorar. & 0,52 & $-0,20$ & 0,38 & 0,38 & 0,40 & 0,34 & 0,52 & 0,25 & 0,33 & 0,05 & 0,50 & 0,11 \\
\hline $\begin{array}{l}\text { 4. Me gustaría ser feliz como las otras } \\
\text { personas. }\end{array}$ & 0,40 & $-0,10$ & 0,31 & 0,48 & 0,25 & 0,41 & 0,40 & 0,39 & 0,17 & $-0,04$ & 0,50 & $-0,04$ \\
\hline 12. No tengo confianza en mí mismo(a). & 0,51 & $-0,21$ & 0,49 & 0,35 & 0,30 & 0,27 & 0,56 & 0,20 & 0,23 & 0,11 & 0,50 & 0,38 \\
\hline 2. Me canso con facilidad. & 0,50 & $-0,18$ & 0,23 & 0,40 & 0,24 & 0,31 & 0,35 & 0,25 & 0,27 & 0,24 & 0,39 & 0,01 \\
\hline 1. Me siento bien. & $-0,22$ & 0,58 & 0,01 & 0,76 & 0,13 & 0,64 & 0,25 & 0,72 & 0,01 & 0,46 & 0,14 & 0,63 \\
\hline 10. Soy feliz. & $-0,22$ & 0,63 & 0,04 & 0,80 & 0,06 & 0,61 & 0,15 & 0,82 & $-0,04$ & 0,60 & 0,02 & 0,59 \\
\hline 16. Estoy satisfecho(a). & $-0,19$ & 0,56 & 0,16 & 0,78 & 0,11 & 0,69 & 0,33 & 0,63 & 0,13 & 0,55 & 0,25 & 0,57 \\
\hline $\begin{array}{l}\text { 7. Soy tranquilo(a), equilibrado(a) y } \\
\text { dueño(a) de mí mismo(a). }\end{array}$ & $-0,22$ & 0,56 & 0,24 & 0,63 & 0,23 & 0,44 & 0,49 & 0,22 & 0,06 & 0,30 & 0,23 & 0,53 \\
\hline 19. Soy una persona estable. & $-0,20$ & 0,59 & 0,23 & 0,63 & 0,16 & 0,54 & 0,52 & 0,29 & 0,12 & 0,39 & 0,43 & 0,53 \\
\hline 13. Me siento seguro(a). & $-0,26$ & 0,68 & 0,34 & 0,60 & 0,26 & 0,52 & 0,59 & 0,34 & 0,11 & 0,61 & 0,26 & 0,52 \\
\hline 14. Evito enfrentar dificultades. & 0,33 & 0,04 & 0,42 & $-0,01$ & 0,16 & 0,01 & 0,03 & $-0,05$ & 0,19 & 0,04 & 0,39 & $-0,49$ \\
\hline 6. Me siento descansado(a). & $-0,15$ & 0,34 & 0,13 & 0,47 & 0,11 & 0,45 & 0,45 & 0,30 & 0,13 & 0,23 & 0,26 & 0,38 \\
\hline
\end{tabular}

* Tabla adaptada del estudio de Fioravanti et. al. (2006).

** Indica que el orden de los factores cambia. 
utilizados en la evaluación de deportistas y atletas en Brasil. De hecho, la muestra de esta investigación tiene mayoría de hombres (cerca de 60\%), que es algo que los estudios de los autores que encontraron una estructura de ansiedad presente y ausente tienen en común. De esa forma, puede cuestionarse si esta estructura ocurre en razón de la actividad que realizan, o si es una característica más presente en el sexo masculino.

Se puede observar en este estudio que las cargas factoriales de los ítems del STAI-T son superiores a las cargas factoriales encontradas en otros estudios brasileros que utilizaron muestras diferentes, principalmente en el factor ansiedad presente (Factor 1). Ese hecho sugiere que la práctica de deportes puede favorecer una mayor presencia de características de ansiedad. Sobre ese hecho, es importante destacar que el STAI-T presenta una mayor parte de los ítems relacionados a la ansiedad cognitiva, y que la posibilidad de competitividad produce expectativas y afecta la evaluación de la situación, lo cual, a su vez, puede afectar la ansiedad cognitiva (Jones \& Hanton, 2001; Jones $\&$ Swain, 1992). Ese aspecto también puede estar aumentado por la percepción de amenaza y la retroalimentación del fracaso, comunes en situaciones deportivas (Estrada \& Pérez, 2008) y presentes en buena parte de los ítems del STAI-T. A pesar de estas posibles explicaciones, deben realizarse nuevas investigaciones con el objetivo de identificar los procesos de respuesta de deportistas, así como otros aspectos cognitivos de la ansiedad de este grupo que favorezcan una identificación más clara de sus señales y una distinción de otras expresiones ansiogénicas propias de otros grupos y contextos.

Otro aspecto que debe señalarse es que algunos ítems $(4,6,13,14$ y 19) tuvieron cargas factoriales más altas en factores inversos a las muestras de comparación, o sea, mientras que en los deportistas fueron más altas en el factor 1, en las muestras de universitarios lo fueron en el factor 2. Considerando la muestra de deportistas, aparentemente las mayores discrepancias ocurrieron con el grupo de universitarios, mientras que las mayores semejanzas fueron con el grupo de militares. De hecho, esas semejanzas tal vez puedan ser asociadas al énfasis que el deporte tiene en el servicio militar brasilero. De cualquier modo, ese hecho refuerza la necesidad de que se lleven a cabo investigaciones al respecto, una vez que tal afirmación no puede ser respaldada por los datos de esta investigación, los cuales apenas indican una relación, sin establecer modelos causales.

Es importante destacar que las cargas factoriales visualizadas en las tablas 1 y 2 son diferentes, y que eso ocurre porque para establecer la comparación con los otros estudios se decidió mantener todos los ítems del STAI-T. Pese a eso, se pone de presente que la estructura del instrumento que mejor se ajustó a los deportistas brasileros fue la presentada en la tabla 1, en la cual los peores ítems (con cargas factoriales más bajas) fueron retirados.

\section{Consideraciones finales}

Uno de los factores que motivaron el desarrollo de esta investigación fue el estudio de Brandão (2007), el cual destacó que la mayoría de los instrumentos psicológicos que se utilizan en Brasil para evaluar deportistas no tienen características psicométricas adecuadas para esa población. Adicionalmente, en la literatura no se encontraron estudios que demuestren la estructura factorial del STAI-T en muestras específicas de deportistas brasileros. El análisis factorial realizado proporcionó dos factores que se refieren a la ansiedad presente y a la ansiedad ausente. Este resultado es consistente con el trabajo realizado por Spielberger et. al. (1970) y con la adaptación española realizada por Bermúdez (1977), siendo que el porcentaje de variancia demostrado por Bermúdez fue superior al observado en este estudio. A ese respecto debe ser considerado que los dos estudios mencionados trabajaron con todos los indicadores del STAI, o sea, la ansiedad rasgo y la ansiedad estado, lo que sin duda aumenta la variancia de los datos. El hecho de que esta investigación trabajó apenas con el STAI-T puede tener influencia sobre la variancia observada. En relación con los índices de confiabilidad, los hallazgos de Spielberger et. al. (1970) y Bermúdez (1977) también fueron superiores a los verificados en este estudio.

Es importante que se den algunas sugerencias, como la realización de futuras investigaciones que relacionen los resultados del STAI con otras medidas de ansiedad en deportistas brasileros. Estudios como los de Cattell y Scheier (1963) y Spielberger et. al. (1970) son ejemplos de trabajos que mostraron asociaciones del STAI con otras medidas de ansiedad, caracterizando evidencias de validez convergente (APA, AERA \& NCME, 1999) para el instrumento. Asimismo, es de particular interés para los entrenadores, los atletas y los investigadores de la psicología del deporte, la relación entre la ansiedad y el rendimiento deportivo. Sobre ese aspecto, sería muy importante que futuras investigaciones puedan comparar el resultado obtenido por deportistas con el resultado de atletas de alto rendimiento de diferentes modalidades. El interés por la evaluación de la ansiedad en atletas de alto rendimiento es de gran importancia para investigadores de diversos países (Morgan, O'Connor, Ellickson \& Bradley, 1988; Porat, Lufi \& Tenenbaum, 1989; Schwenkmezger \& Laux, 1986).

Otro aspecto interesante es destacar que sería positivo investigar la unidimensionalidad del instrumento, 
considerando que sus factores aparentemente representan dos polos de una misma dimensión. El Modelo de Rasch (uno de los parámetros derivados del modelo de Teoría de Respuesta al Ítem) sería una posibilidad para ello, ya que permite el estudio de la unidimensionalidad de las pruebas por medio del análisis de componentes principales de los residuos. También sería posible identificar patrones de respuesta a los elementos inesperados, o incluso limitar el número de categorías más adecuadas para la evaluación de los ítems. Finalmente, sería importante considerar si ítems como "soy feliz" o "estoy satisfecho" son de hecho aspectos opuestos a la ansiedad, y si nuevos estudios mantendrían esos ítems en la estructura aquí observada. Se espera que este trabajo sirva como un motivador para que se realicen otras investigaciones que puedan contribuir con el trabajo práctico del psicólogo del deporte, así como la realización de diagnósticos de ansiedad más eficientes por medio del STAI-T.

\section{REFERENCIAS}

Abenza, L., Alarcón, F., Leite, N., Ureña, N., \& Piñar, M. (2009). Relación entre la ansiedad y la eficacia de un equipo de baloncesto durante la competición. Cuadernos de Psicología del Deporte, 9 (suplementario), 51.

American Educational Research Association, American Psychological Association, \& National Council on Measurement in Education (1999). Standards for educational and psychological testing. Washington, DC. American Psychological Association.

Aguirre-Loaiza, H. H., \& Bermúdez, S. R. (2011). Ansiedadestado y variables sociodemográficas en futbolistas juveniles colombianos durante competencia. Diversitas: Perspectivas en Psicología, 7(2), 239-251.

Andrade, L., Gorenstein, C., Vieira, A. H., Tung, T. C., \& Artes, R. (2001). Psychometric properties of the Portuguese version of the State-Trait Anxiety Inventory applied to college students: Factor analysis and relation to the Beck depression Inventory. Brazilian Journal of Medical and Biological Research, 34, 367-374.

Arbuckle, J. L., \& Worthke, W. (1999). AMOS 4.0 users guide. Chicago: SmallWaters.

Bartholomeu, D., Machado, A. A., Spigato, F., Bartholomeu, L. L., Cozza, H. P., \& Montiel, J. M. (2009). Traços de personalidade, ansiedade e depressão em jogadores de futebol. Revista brasileira de psicologia do esporte, 3(4), 98-114.

Bermúdez, J. (1977). Ansiedad y rendimiento. Tesis doctoral. Universidad Complutense de Madrid, Madrid, España.

Brandão, M. R. F. (2007). A psicologia do exercício e do esporte e seus desafios para o milênio. En M. R. F. Brandão \& A. A. Machado (Eds.). Coleção Psicologia do esporte e do exercício: Teoria e aplicação. Atheneu: São Paulo.
Cattell, R. B., \& Scheier, I. H. (1963). Handbook for the IPAT Anxiety Scale Questionnaire. Champaign, IL: Institute for Personality and Ability Testing.

Cattell, R. B. (1966). The meaning and strategic use of factor analysis. En R. B. Cattell (Ed.), Handbook of multivariate experimental psychology (pp. 174-243). Chicago: Rand McNally.

Estrada, C. O., \& Pérez, C. E. (2008). Palabras e imágenes positivas en la respuesta de ansiedad en deportistas de competición. Cuadernos de Psicología del Deporte, 8(1), 31-45.

Fioravanti, A. C. M., Santos, L. F., Maissonette, S., Cruz, A. P. M., \& Landeira-Fernandez, J. (2006). Avaliação da estrutura fatorial da escala de ansiedade-traço do IDATE. Avaliação Psicológica, 5(2), 217-224.

Gillet, N., Rosnet, E., \& Vallerand, R. J. (2008). Development of a scale of satisfaction of the fundamental requirements in sporting context. Canadian Journal of Behavioural Science/Revue canadienne des sciences du comportement, 40(4), 230-237.

Gonçalves, M. G., \& Belo, R. P. (2007). Ansiedade Traço Competitiva: diferenças quanto ao gênero, faixa etária, experiência em competições e modalidade esportiva em atletas. Psico-USF, 12(2), 301-307.

Guilford, J. P., \& Fruchter, B. (1978). Fundamental statistics in psychology and education. New York: McGraw-Hill.

Hackfort, D., \& Spielberger, C. (1989). Anxiety in sports: an international perspective. New York: Hemisphere Publishing.

Hair, J. F., Anderson, R. E., Tatham, R. L., \& Black, W. C. (1995). Multivariate Data analysis with readings. New Jersey: Prentice-hall international.

Hayton, J. C, Allen, D. G., \& Scarpello, V.G. (2004) "Factor Retention Decisions in Exploratory or Analysis: A Tutorial on Parallel Analysis." Organizational Research Methods, 7(2), 191-205.

Jones, G., \& Hanton, S. (2001). Pre-competitive feeling states and directional anxiety interpretations. Journal of Sport Sciences, 19, 385-395.

Jones, G., \& Swain, A. B. J. (1992). Intensity and direction dimensions of competitive state anxiety and relationships with competitiveness. Perceptual and Motor Skills, 74, 467472.

Kennedy, B. L., Schwab, J. J., Morris, R. L., \& Beldia, G. (2001). Assessment of State and Trait Anxiety in Subjects with Anxiety and Depressive Disorders. Psychiatric Quarterly, 72(3), 263-276.

MacCallum, R. C., Widaman, K. F., Zhang, S., \& Hong, S. (1999). Sample size in factoranalysis. Psychological Methods, 4(1), 84-99.

Moreno, D. A. C., \& Copete, P. E. B. (2005). Validación del inventario de ansiedad estado-rasgo (STAIC) en niños escolarizados entre los 8 y 15 años. Acta Colombiana de Psicología, 13(5), 79-90. 
Morgan, W. P., O’Connor, P. J., Ellickson, K. A., \& Bradley, P. W. (1988). Personality structure, mood states and performance in elite male distance runners. International Journal of Sport Psychology, 19, 247-263.

Pasquali, L., Pinelli Jr, B., \& Solha, A. C. (1994). Contribuição à validade e normatização da escala de ansiedade traçoestado do IDATE. Psicologia: Teoria e Pesquisa, 10(3), 411-420.

Pesca, A. D. (2004). Intervenção Psicológica em um Trabalho Interdisciplinar na Recuperação de Atletas Lesionados de Futebol. Tesina de Maestría. Universidade Federal de Santa Catarina, Florianópolis, SC, Brasil.

Porat, Y., Lufi, D., \& Teenbaum, G. (1989). Psychological components contribute to select young female gymnasts. International Journal of Sport Psychology, 20(4), 279-286.

Schwenkmezger, P., \& Laux, L. (1986). Trait anxiety, worry, and emotionality in athletic competition. En C. D. Spielberger, \& R. Diaz-Guerrero (Eds.), Cross-cultural anxiety (p. 3). Washington, DC: Hemisphere.

Serrato, L. H, García, S. Y., \& Rivera, J. F. (2000). Baremos de la prueba STAI para un grupo de deportistas bogotanos (Manual). Bogotá: PAR LTDA.
Smith, R. E., Smoll, F. L., \& Wiechman, S. A. (1999). Measurement of trait anxiety in sport. En J. L. Duda (Ed.). Advances in sport and exercise psychology measurement. Morgan Town: F.I.T. Inc.

Spielberger, C. D., Gorsuch, R.L., \& Lushene. R. E. (1970). Manual for the State-Trait Anxiety Inventory. Palo Alto, CA: Consulting Psychologists Press.

Tabachinick, B. G., \& Fidell, L. S. (1996). Using multivariate statistics. New York: HarperCollins.

Urraca, S. (1981). Actitudes ante la muerte (preocupación, ansiedad, temor) y religiosidad. Tesis doctoral. Facultad de Psicología. Universidad Complutense de Madrid, Madrid, España.

Wamba, C., Vicari, C., Elisenhut, R. D., Radtke, C. L., \& Shigunov, V. (2008). Ansiedade Pré-Competitiva: O Caso do Hockey na Grama. Trabajo presentado en el XII Congresso Paulista de Educação Física. Jundiaí, San Pablo, Brasil. 